九州大学学術情報リポジトリ

Kyushu University Institutional Repository

\title{
Cyclic Classes and an Ergodic Theorem in Dynamic Fuzzy Systems
}

Yoshida, Yuj i

Faculty of Economics and Business Administration, Kitakyushu University

http://hdl. handle. net/2324/3191

出版情報 : RIFIS Technical Report. 101，1995-02. Research Institute of Fundamental Information Science, Kyushu University バージョン：

権利関係 : 
RIFIS-TR-CS-101

\section{RIFIS Technical Report}

Cyclic Classes and an Ergodic Theorem

in Dynamic Fuzzy Systems

Y. Yoshida

February ,1995

Research Institute of Fundamental Information Science Kyushu University 33

Fukuoka 812, Japan

E-mail: Phone: 


\title{
CYCLIC CLASSES AND AN ERGODIC THEOREM IN DYNAMIC FUZZY SYSTEMS
}

\author{
Yuji YOSHIDA \\ Faculty of Economics and Business Administration, Kitakyushu University, \\ 4-2-1 Kitagata, Kokuraminami, Kitakyushu 802, Japan
}

\begin{abstract}
This paper analyses a recurrent behavior of dynamic fuzzy systems defined by fuzzy relations on a metric space. We show the existence of cyclic classes of the dynamic fuzzy system and give an ergodic theorem for the fuzzy transition.
\end{abstract}

Keyword : Cyclic class; ergodic theorem; dynamic fuzzy system; recurrence; fuzzy relation.

\section{Introduction and notations}

Limit theorems of a sequence of fuzzy sets defined successively by fuzzy relations are first studied by Bellman and Zadeh [1]. Kurano et al. [3], under a contraction condition, studied the limiting behavior of fuzzy states defined by the dynamic fuzzy system with a compact space. A notion of recurrent sets for the dynamic fuzzy system is given by Yoshida [7]. This paper analyses the cyclic behavior of the dynamic fuzzy system.

In Section 2, we show the existence of cyclic classes, which are nested with respect to levels, for recurrent sets in the dynamic fuzzy system, using the methods of Chung [2]. In Section 3, we show a correspondence between a period of the cyclic classes and a limiting possibility of the fuzzy transition. By the use of the result, we give an ergodic theorem which is different from the ergodicity in the classical Markov chains. In the dynamic fuzzy system, the limit of the fuzzy transition equals to one of a finite/countable number of levels, and the states are classified into cyclic classes nested with respect to the levels. In Section 4, a numerical example is given to illustrate our idea.

\section{Cyclic classes for dynamic fuzzy systems}

Let a state space $E$ be a complete metric space. Let a time space by $\mathbf{N}:=\{0,1,2, \cdots\}$. Let $\tilde{q}$ be an upper semi-continuous fuzzy relation on $E \times E$ satisfying the following normality condition :

$$
\sup _{x \in E} \tilde{q}(x, y)=1(y \in E) \text { and } \sup _{y \in E} \tilde{q}(x, y)=1(x \in E) \text {. }
$$

We denote a fuzzy set on $E$ by its membership function $\tilde{s}: E \mapsto[0,1]$ ([3]). Kurano et al. [3], under a contractive condition, studied the limiting behavior of a sequence of fuzzy 
states $\left\{\tilde{s}_{n}\right\}_{n=0}^{\infty}$ given as follows : An initial fuzzy state $\tilde{s}_{0}$ is a given fuzzy set on $E$, and we define

$$
\tilde{s}_{n+1}(y):=\sup _{x \in E}\left\{\tilde{s}_{n}(x) \wedge \tilde{q}(x, y)\right\}, \quad y \in E, n=0,1,2, \cdots .
$$

This is called a dynamic fuzzy system (see $[3,6,8,9]$ ). We define a sequence of fuzzy relations, $\left\{\tilde{q}^{n}\right\}_{n=0}^{\infty}$, on $E \times E$ as follows : For $x, y \in E$,

$$
\begin{aligned}
& \tilde{q}^{0}(x, y):=\left\{\begin{array}{ll}
1 & \text { if } x=y, \\
0 & \text { if } x \neq y ;
\end{array} \quad \tilde{q}^{1}(x, y):=\tilde{q}(x, y), \quad\right. \text { and } \\
& \tilde{q}^{n+1}(x, y):=\sup _{z \in E}\left\{\tilde{q}^{n}(x, z) \wedge \tilde{q}(z, y)\right\} \quad n=1,2,3, \cdots,
\end{aligned}
$$

where the operation $\wedge$ means that $a \wedge b=\min \{a, b\}$ for real numbers $a$ and $b$. Then, $\tilde{q}^{n}(x, y)$ means the possibility to transit from a point $x$ to a point $y$ at the $n$th step. The sequence of fuzzy state $\left\{\tilde{s}_{n}\right\}_{n=0}^{\infty}$ in $(2.1)$ can be written as

$$
\tilde{s}_{n}(y)=\sup _{x \in E}\left\{\tilde{s}_{0}(x) \wedge \tilde{q}^{n}(x, y)\right\}, \quad y \in E, n=0,1,2, \cdots .
$$

In this paper, we discuss the cyclic property of the fuzzy relations $\left\{\tilde{q}^{n}(x, y)\right\}_{n=0}^{\infty}$.

Let $x, y \in E$. For $\alpha \in(0,1]$, we write

$$
x \underset{\alpha}{\sim} y \text { if there exists a positive integer } n \text { such that } \hat{q}^{n}(x, y) \geq \alpha .
$$

We also write

$$
\underset{0}{\sim} y \text { if there exists a positive integer } n \text { such that } \hat{q}^{n}(x, y)>0
$$

Definition 2.1. For $\alpha \in[0,1]$, we call a non-empty set $A(\subset E) \alpha$-essential if

$$
x \underset{\alpha}{\sim} y \text { and } \underset{\alpha}{\sim} x \quad \text { for all } x, y \in A .
$$

We define $A_{\alpha}(x):=\left\{y \in E \mid x \sim_{\alpha} y\right.$ and $\left.y \sim_{\alpha} x\right\}$ for $x \in E$ and $\alpha \in[0,1] . A_{\alpha}(x)$ is clearly $\alpha$-essential if it is not empty. Next we define

$$
\mathbf{I}(x, \alpha):= \begin{cases}\left\{\text { positive integers } n \mid \tilde{q}^{n}(x, x) \geq \alpha\right\} & \text { for } x \in E, \alpha>0 \\ \left\{\text { positive integers } n \mid \hat{q}^{n}(x, x)>0\right\} & \text { for } x \in E, \alpha=0 .\end{cases}
$$

Then we put $d_{x, \alpha}:=\operatorname{gcd} \mathbf{I}(x, \alpha)(x \in E, \alpha \in[0,1])$, where gcd means the greatest common divisor of a set of positive integers. We call $d_{x, \alpha}$ a period of a state $x$ with a level $\alpha$. The following lemmas hold for $\alpha \in[0,1]$ and $x \in E$ such that $\mathbf{I}(x, \alpha) \neq \emptyset$.

\section{Lemma 2.1.}

(i) If $m, n \in \mathbf{I}(x, \alpha)$, then $m+n \in \mathbf{I}(x, \alpha)$. 
(ii) If $m \in \mathbb{I}(x, \alpha)$, then $m n \in \mathbb{I}(x, \alpha)$ for all positive integers $n$.

Proof. (i) Let $\alpha>0$ and $m, n \in \mathrm{I}(x, \alpha)$. Then

$$
\hat{q}^{m+n}(x, x)=\sup _{y \in E}\left\{\hat{q}^{m}(x, y) \wedge \hat{q}^{n}(y, x)\right\} \geq \hat{q}^{m}(x, x) \wedge \hat{q}^{n}(x, x) \geq \alpha .
$$

Therefore $m+n \in \mathbf{I}(x, \alpha)$ and so (i) holds. We can easily check the case of $\alpha=0$ similarly. (ii) is trivial from (i).

Lemma 2.2. A family of periods, $\left\{d_{x, \alpha} \mid \alpha \in[0,1]\right\}$, has the following properties:

(i) $d_{x, \alpha^{\prime}}$ divides $d_{x, \alpha}$ if $0 \leq \alpha^{\prime}<\alpha \leq 1$,

(ii) the map $\alpha(\in(0,1]) \mapsto d_{x, \alpha}(\in\{1,2,3, \cdots\})$ is a non-decreasing left-continuous piecewise constant function.

Proof. (i) Let $\alpha, \alpha^{\prime}$ satisfy $0 \leq \alpha^{\prime}<\alpha \leq 1$. Since $\mathbf{I}\left(x, \alpha^{\prime}\right) \supset \mathrm{I}(x, \alpha)$, we have

$\left\{d \mid d\right.$ is a common devisor of $\left.\mathbf{I}\left(x, \alpha^{\prime}\right)\right\} \subset\{d \mid d$ is a common devisor of $\mathbf{I}(x, \alpha)\}$.

Therefore $d_{x, \alpha^{\prime}} \leq d_{x, \alpha}$, and so $d_{x, \alpha^{\prime}}$ divides $d_{x, \alpha}$.

(ii) From the definition of $\mathbb{I}(x, \alpha)$, we have

$$
\lim _{\alpha^{\prime} \uparrow \alpha} \mathbf{I}\left(x, \alpha^{\prime}\right)=\bigcap_{\alpha^{\prime}<\alpha} \mathbf{I}\left(x, \alpha^{\prime}\right)=\mathbf{I}(x, \alpha) .
$$

Therefore $\lim _{\alpha^{\prime} \uparrow \alpha} d_{x, \alpha^{\prime}}=d_{x, \alpha}$. We obtain the assertion (ii) since the map $\alpha \mapsto d_{x, \alpha}$ takes discrete values.

We prepare several lemmas, referring [2, Theorems I.3.2 and I.3.3], to show the existence of cyclic classes for the dynamic fuzzy system.

Lemma 2.3. If $x \sim_{\alpha} y$ and $y \sim_{\alpha} x$, then $d_{x, \alpha}=d_{y, \alpha}$.

Proof. Let $\alpha>0$. Since $x \sim_{\alpha} y$ and $y \sim_{\alpha} x$, there exist positive integers $m$ and $n$ such that $\tilde{q}^{m}(x, y) \geq \alpha$ and $\tilde{q}^{n}(y, x) \geq \alpha$. If $l \in \mathbf{I}(x, \alpha)$, then

$$
\tilde{q}^{n+l+m}(y, y) \geq \tilde{q}^{n}(y, x) \wedge \tilde{q}^{l}(x, x) \wedge \tilde{q}^{m}(x, y) \geq \alpha .
$$

Therefore $n+l+m \in \mathbf{I}(y, \alpha)$. Since $\tilde{q}^{2 l}(x, x) \geq \tilde{q}^{l}(x, x) \wedge \tilde{q}^{l}(x, x) \geq \alpha$, we also have $n+2 l+m \in \mathbf{I}(y, \alpha)$. So we obtain $l=(n+2 l+m)-(n+l+m) \equiv 0\left(\bmod d_{y, \alpha}\right)$ for all $l \in \mathbf{I}(x, \alpha)$. Therefore $d_{y, \alpha}$ divides $d_{x, \alpha}$. Similarly we can prove that $d_{x, \alpha}$ divides $d_{y, \alpha}$. Thus we obtain $d_{x, \alpha}=d_{y, \alpha}$. We can check the case of $\alpha=0$ similarly.

Lemma 2.4. Let $\alpha>0$ and $y \in A_{\alpha}(x)$. Then there exists a unique integer $n_{x, y, \alpha}$ $\left(0 \leq n_{x, y, \alpha}<d_{x, \alpha}\right)$ satisfying the following (i) : 
(i) if $\tilde{q}^{m}(x, y) \geq \alpha$, then $m \equiv n_{x, y, \alpha}\left(\bmod d_{x, \alpha}\right)$.

(ii) Further, there exists a positive integer $N_{x, y, \alpha}$ such that $\hat{q}^{n d_{x, \alpha}+n_{x, y, \alpha}}(x, y) \geq \alpha$ for all $n \geq N_{x, y, \alpha}$.

Proof. (i) Let positive integers $m$ and $n$ satisfy $\hat{q}^{m}(x, y) \geq \alpha$ and $\tilde{q}^{n}(x, y) \geq \alpha$. Let a positive integer $l$ satisfy $\tilde{q}^{l}(y, x) \geq \alpha$. Then $\tilde{q}^{m+l}(x, x) \geq \tilde{q}^{m}(x, y) \wedge \tilde{q}^{l}(y, x) \geq \alpha$. Similarly $\tilde{q}^{n+l}(x, x) \geq \tilde{q}^{n}(x, y) \wedge \tilde{q}^{l}(y, x) \geq \alpha$. Therefore $m \equiv n\left(\bmod d_{x, \alpha}\right)$. Thus we obtain (i).

(ii) Let $n_{i} \in \mathbf{I}(x, \alpha)(i=1,2, \cdots, k)$. From an elementary result of number theory, there exists $N_{x, \alpha}^{\prime}$ such that for each positive integer $n\left(n \geq N_{x, \alpha}^{\prime}\right)$ there exist positive integers $c_{i}(i=1,2, \cdots, k)$ satisfying

$$
n d_{x, \alpha}=\sum_{i=1}^{k} c_{i} n_{i} .
$$

Then, from Lemma 2.1,

$$
\hat{q}^{n d_{x, \alpha}}(x, x) \geq \bigwedge_{i=1,2, \cdots, k} \tilde{q}^{c_{i} n_{i}}(x, x) \geq \alpha
$$

From (i), there exists a positive integer $l$ such that $\tilde{q}^{l_{x, \alpha}+n_{x, y, \alpha}}(x, y) \geq \alpha$. Put $N_{x, y, \alpha}:=$ $N_{x, \alpha}^{\prime}+l$. For all $n \geq N_{x, y, \alpha}$, we obtain

$$
\hat{q}^{n d_{x, \alpha}+n_{x, y, \alpha}}(x, y) \geq \tilde{q}^{n^{\prime} d_{x, \alpha}}(x, x) \wedge \hat{q}^{m d_{x, \alpha}+n_{x, y, \alpha}}(x, y) \geq \alpha
$$

where $n^{\prime}:=n-l \geq N_{x, \alpha}^{\prime}$. Thus we get (ii).

We also have similar results for the case of $\alpha=0$, which can be checked in the same way as Lemma 2.4 .

Corollary 2.1. Let $y \in A_{0}(x)$. Then there exists a unique integer $n_{x, y, 0}\left(0 \leq n_{x, y, 0}<\right.$ $\left.d_{x, 0}\right)$ satisfying the following (i) :

(i) if $\tilde{q}^{m}(x, y)>0$, then $m \equiv n_{x, y, 0}\left(\bmod d_{x, 0}\right)$.

(ii) Further there exists a positive integer $N_{x, y, 0}$ such that $\tilde{q}^{n d_{x, 0}+n_{x, y, 0}}(x, y)>0$ for all $n \geq N_{x, y, 0}$.

Let $\alpha \in[0,1]$ and $x \in E$ satisfy $\mathbf{I}(x, \alpha) \neq \emptyset$. For a positive integer $d$, we put subsets of $A_{\alpha}(x)$ by

$D_{\alpha, m}(x):= \begin{cases}\left\{y \in A_{\alpha}(x) \mid \tilde{q}^{n}(x, y) \geq \alpha \text { for some } n \text { satisfying } n \equiv m(\bmod d)\right\} & \text { if } \alpha>0, \\ \left\{y \in A_{0}(x) \mid \tilde{q}^{n}(x, y)>0 \text { for some } n \text { satisfying } n \equiv m(\bmod d)\right\} & \text { if } \alpha=0,\end{cases}$ 
for $m=0,1,2 \cdots, d-1$. If $\left\{D_{\alpha, n}(x)\right\}_{n=0,1,2 \cdots, d-1}$ are disjoint and their union equals to $A_{\alpha}(x)$, then they are called subclasses of $A_{\alpha}(x)$ with the period $d$ (c.f. [2, Section I.3]). For convenience, for $n \in \mathrm{N}$, we put

$$
D_{\alpha, n}(x):=D_{\alpha, m}(x) \text { if } n \equiv m(\bmod d) .
$$

Now we define subsets of $A_{\alpha}(x)$ by

$C_{\alpha, m}(x):= \begin{cases}\left\{y \in A_{\alpha}(x) \mid \hat{q}^{n}(x, y) \geq \alpha \text { for some } n \text { satisfying } n \equiv m\left(\bmod d_{x, \alpha}\right)\right\} & \text { if } \alpha>0 \\ \left\{y \in A_{0}(x) \mid \tilde{q}^{n}(x, y)>0 \text { for some } n \text { satisfying } n \equiv m\left(\bmod d_{x, 0}\right)\right\} & \text { if } \alpha=0\end{cases}$

for $m=0,1,2 \cdots, d_{x, \alpha}-1$. From Lemma 2.4(i) and Corollary 2.1(i), $\left\{C_{\alpha, n}(x)\right\}_{n=0,1,2 \cdots, d_{x, \alpha}-1}$ are subclasses of $A_{\alpha}(x)$ with the period $d_{x, \alpha}$.

We investigate a cyclic property for the dynamic fuzzy system (c.f. [2, Theorem I.3.4]). We use the following notations (see [3]) :

$$
\tilde{q}_{\alpha}(x):=\{y \in E \mid \tilde{q}(x, y) \geq \alpha\} \text { for } x \in E \text { and } \alpha \in(0,1] .
$$

For $\alpha \in(0,1]$ and $x \in E$, we define a sequence of $\left\{\tilde{q}_{\alpha}^{n}(x)\right\}_{n=1}^{\infty}$ by

$$
\tilde{q}_{\alpha}^{1}(x):=\tilde{q}_{\alpha}(x) \text { and } \quad \tilde{q}_{\alpha}^{n+1}(x):=\bigcup_{y \in \tilde{q}_{\alpha}^{n}(x)} \tilde{q}_{\alpha}(y) \text { for } n=0,1,2, \cdots
$$

Definition 2.2 (c.f. [2]). For $\alpha \in(0,1]$, we call a non-empty set $A(\subset E) \alpha$-closed if $\tilde{q}_{\alpha}(x) \subset A$ for all $x \in A$.

Definition 2.3 (c.f. $[2,4])$. Subclasses $D_{\alpha, m}(m=0,1,2, \cdots, d-1)$ with a period $d$ are called cyclic classes if

$$
\tilde{q}_{\alpha}^{n}(y) \subset D_{\alpha, m+n} \quad \text { for all } m=0,1,2 \cdots, d-1 ; n=1,2,3 \cdots ; y \in D_{\alpha, m}
$$

\section{Theorem 2.1.}

(i) If $y \in A_{\alpha}(x)$, then $A_{\alpha}(y)=A_{\alpha}(x)$ and $d_{y, \alpha}=d_{x, \alpha}$.

(ii) Let $m, n \in \mathrm{N}$. If $y \in C_{\alpha, m}(x)$, then $C_{\alpha, n}(y)=C_{\alpha, n+m}(x)$.

(iii) If $A_{\alpha}(x)$ is $\alpha$-closed, then $C_{\alpha, m}(x)\left(m=0,1,2 \cdots, d_{x, \alpha}-1\right)$ are cyclic classes.

Proof. (i) Let $y \in A_{\alpha}(x)$. Let $z \in A_{\alpha}(y)$. Since

$$
x \underset{\alpha}{\sim} \underset{\alpha}{\sim} z \text { and } z \underset{\alpha}{\sim} y \underset{\alpha}{\sim} x
$$

we obtain $z \in A_{\alpha}(x)$. Thus $A_{\alpha}(y) \subset A_{\alpha}(x)$. We obtain $A_{\alpha}(y)=A_{\alpha}(x)$ since the reverse inclusion can be checked similarly. We get (i) together with Lemma 2.3. 
(ii) Let $y \in C_{\alpha, m}(x)$. Then we have

$$
y \in A_{\alpha}(x) \quad \text { and } \quad \tilde{q}^{m^{\prime}}(x, y) \geq \alpha \text { for some } m^{\prime} \text { satisfying } m^{\prime} \equiv m\left(\bmod d_{x, \alpha}\right) \text {. }
$$

For any $z \in C_{\alpha, n}(y)$, we have

$$
z \in A_{\alpha}(y) \text { and } \quad \tilde{q}^{n^{\prime}}(y, z) \geq \alpha \text { for some } n^{\prime} \text { satisfying } n^{\prime} \equiv n\left(\bmod d_{y, \alpha}\right) .
$$

By (i), this yields

$$
z \in A_{\alpha}(x), \quad \hat{q}^{n^{\prime}}(y, z) \geq \alpha \quad \text { and } \quad n^{\prime} \equiv n\left(\bmod d_{x, \alpha}\right) .
$$

Put $l:=m^{\prime}+m^{\prime}$. Then $l \equiv n+m\left(\bmod d_{x, \alpha}\right)$. From $(2.7)$ and $(2.8)$,

$$
\tilde{q}^{l}(x, z)=\tilde{q}^{n^{\prime}+m^{\prime}}(x, z) \geq \tilde{q}^{m^{\prime}}(x, y) \wedge \tilde{q}^{n^{\prime}}(y, z) \geq \alpha .
$$

We obtain $z \in C_{\alpha, n+m}(x)$. Thus we get $C_{\alpha, n}(y) \subset C_{\alpha, n+m}(x)$. Further, since

$$
\bigcup_{n=0,1,2 \cdots, d_{y, \alpha}-1} C_{\alpha, n}(y)=A_{\alpha}(y)=A_{\alpha}(x)=\bigcup_{n=0,1,2 \cdots, d_{x, \alpha}-1} C_{\alpha, n+m}(x),
$$

we get (ii). We can prove the case of $\alpha=0$ similarly.

(iii) Let $m=0,1,2 \cdots, d-1, n=1,2,3 \cdots$ and $y \in C_{\alpha, m}(x)$. Let $z \in \tilde{q}_{\alpha}^{n}(y)$. It is sufficient to check $z \in C_{\alpha, m+n}(x)$. Since $y \in A_{\alpha}(x)$ and $A_{\alpha}(x)$ is $\alpha$-closed,

$$
z \in \tilde{q}_{\alpha}^{n}(y) \subset A_{\alpha}(x)
$$

Since $\tilde{q}^{n}(y, z) \geq \alpha$ from $z \in \tilde{q}_{\alpha}^{n}(y),(2.9)$ and (ii) yield

$$
z \in C_{\alpha, n}(y)=C_{\alpha, n+m}(x)
$$

Therefore we get (iii).

From Theorem 2.1, $\left\{C_{\alpha, n}(x)\right\}_{n=0,1,2 \cdots, d_{x, \alpha}-1}$ does not depend on the choice of $x$. Therefore we denote it by $\left\{C_{\alpha, n}\right\}_{n=0,1,2 \cdots, d_{\alpha}-1}$.

Lemma 2.5. Let $x \in E$. If $\left\{D_{\alpha, m}\right\}_{m=0,1,2, \cdots, d-1}$ be subclasses of $A_{\alpha}(x)$ with a period $d$, then $d$ divides $d_{\alpha}$.

Proof. Let $\alpha \in(0,1]$ and let $m \in \mathbf{I}(x, \alpha)$. Then $\tilde{q}^{m}(x, x) \geq \alpha$. Since $\left\{D_{\alpha, m}\right\}_{m=0,1,2, \cdots, d-1}$ are subclasses of $A_{\alpha}(x)$,

$$
D_{\alpha, 0}=\left\{y \in A_{\alpha}(x) \mid \tilde{q}^{n}(x, y) \geq \alpha \text { for some } n \text { satisfying } n \equiv 0(\bmod d)\right\} .
$$

By renumbering $\left\{D_{\alpha, m}\right\}_{m=0,1,2, \cdots, d-1}$ if necessary, we may assume that $C_{\alpha, 0} \cap D_{\alpha, 0} \neq \emptyset$. For $y \in C_{\alpha, 0} \cap D_{\alpha, 0}$, there exists $n$ such that

$$
\tilde{q}^{n}(x, y) \geq \alpha \quad \text { and } \quad n \equiv 0(\bmod d) .
$$


Therefore $\hat{q}^{m+n}(x, y) \geq \hat{q}^{m}(x, x) \wedge \tilde{q}^{n}(x, y) \geq \alpha$. We have $m+n \equiv 0$ (mod $\left.d\right)$ since $\left\{D_{\alpha, l}\right\}_{l=0,1,2, \cdots, d-1}$ are disjoint. Together with $(2.10)$, we obtain $m \equiv 0(\bmod d)$. Namely $d$ divides all $m \in \mathbf{I}(x, \alpha)$. Thus $d$ divides $d_{\alpha}$. We can check the case of $\alpha=0$ similarly.

Theorem 2.2. Let $A_{\alpha}(x)$ be $\alpha$-closed. Let $\left\{D_{\alpha, m}\right\}_{m=0,1,2, \cdots, d-1}$ be subclasses of $A_{\alpha}(x)$ with a period $d$. If $\left\{D_{\alpha, m}\right\}_{m=0,1,2, \cdots, d-1}$ are cyclic classes, then each $D_{\alpha, m}$ is the union of $d_{\alpha} / d$ sets from $\left\{C_{\alpha, n}\right\}_{n=0,1,2, \cdots, d_{\alpha}-1}$.

Proof. With no loss of generality, we may assume that $x \in C_{\alpha, 0} \cap D_{\alpha, 0}$. Then $\left\{C_{\alpha, n} \cap\right.$ $\left.D_{\alpha, m}\right\}_{n=0,1,2, \cdots, d_{\alpha}-1 ; m=0,1,2, \cdots, d-1}$ are subclasses of $A_{\alpha}(x)$ with a period $d_{\alpha} d$. Suppose that $C_{\alpha, m^{\prime}} \cap D_{\alpha, m^{\prime}} \neq C_{\alpha, m^{\prime}}$ for some $m^{\prime}$. Let $y \in C_{\alpha, m^{\prime}} \backslash\left(C_{\alpha, m^{\prime}} \cap D_{\alpha, m^{\prime}}\right)$. Since $y \notin\left(C_{\alpha, m^{\prime}} \cap D_{\alpha, m^{\prime}}\right)$, we have

$$
\tilde{q}^{l d_{\alpha} d+m^{\prime}}(x, y)<\alpha \text { for all } l=1,2, \cdots .
$$

This contradicts $y \in C_{\alpha, m^{\prime}}$. Therefore we obtain $C_{\alpha, m} \subset D_{\alpha, m}$ for all $m=0,1, \cdots, d-1$. Using the cyclic assumption for $\left\{D_{\alpha, m}\right\}_{m=0,1,2, \cdots, d-1}$, we have $C_{\alpha, m} \subset D_{\alpha, m}=D_{\alpha, n}$ if $0 \leq n<d_{\alpha}$ and $n \equiv m(\bmod d)$. Therefore $\bigcup_{l=0,1,2, \cdots, n^{\prime}-1} C_{\alpha, l d+m} \subset D_{\alpha, m}$, where we put a positive integer $n^{\prime}:=d_{\alpha} / d$ from Lemma 2.5. Since

$$
\bigcup_{m=0,1,2, \cdots, d-1} \bigcup_{l=0,1,2, \cdots, n^{\prime}-1} C_{\alpha, l d+m}=A_{\alpha}=\bigcup_{m=0,1,2, \cdots, d-1} D_{\alpha, m l},
$$

we obtain $D_{\alpha, m}=\bigcup_{l=0,1,2, \cdots, n^{\prime}-1} C_{\alpha, l d+m}$ for $m=0,1,2, \cdots, d-1$.

\section{An ergodic theorem}

In this section, we consider the periodic limit of the sequence of the fuzzy transitions, $\left\{\tilde{q}^{n}(x, y)\right\}_{n=0}^{\infty}$, and discuss the ergodic property (c.f [2, Theorems I.6.1 and I.6.4]). Define

$$
\alpha(x):=\sup _{n \geq 1} \hat{q}^{n}(x, x) \quad \text { for } x \in E .
$$

We put

$$
A^{*}:=\{x \in E \mid \alpha(x)>0\}=\left\{x \in E \mid \sup _{n \geq 1} \hat{q}^{n}(x, x)>0\right\}=\left\{x \in E \mid x \in A_{0}(x)\right\} .
$$

Then, $A^{*}$ equals to the union of all positive recurrent sets (see [7, Theorem 3.1]). By the results of Section 2, we have the following limit theorem on $A^{*}$ for the dynamic fuzzy system.

Theorem 3.1. Let $x \in A^{*}$. Put

$$
d(x):=\lim _{\alpha \uparrow \alpha(x)} \operatorname{gcd}\left\{n \geq 1 \mid \tilde{q}^{n}(x, x) \geq \alpha\right\} .
$$

If $d(x)<\infty$, then we have

$$
\lim _{n \rightarrow \infty} \tilde{q}^{n d(x)}(x, x)=\alpha(x) .
$$


Proof. Let $\alpha$ satisfy $0<\alpha<\alpha(x)$. Then $x \in A_{\alpha}(x)$. Therefore, there exists a period $d_{\alpha}$ of $A_{\alpha}(x)$. Let $\underline{\lambda}_{\alpha}:=\underline{\lim }_{n \rightarrow \infty} \hat{q}^{n d_{\alpha}}(x, x)$ and $\bar{\lambda}_{\alpha}:=\overline{\lim }_{n \rightarrow \infty} \hat{q}^{n d_{\alpha}}(x, x)$. From Lemma 2.4(ii), we have

$$
\alpha \leq \underline{\lambda}_{\alpha} \leq \bar{\lambda}_{\alpha} \leq \sup _{n \geq 1} \hat{q}^{n}(x, x)=\alpha(x)
$$

Therefore

$$
\alpha(x)=\lim _{\alpha \uparrow \alpha(x)} \underline{\lambda}_{\alpha}=\lim _{\alpha \uparrow \alpha(x)} \bar{\lambda}_{\alpha}
$$

From Lemma 2.2(i), there exists a limit

$$
d(x):=\lim _{\alpha \uparrow \alpha(x)} d_{\alpha}=\lim _{\alpha \uparrow \alpha(x)} \operatorname{gcd}\left\{n \geq 1 \mid \tilde{q}^{n}(x, x) \geq \alpha\right\} .
$$

Suppose that $d(x)<\infty$. From (3.3) and Lemma 2.2(ii), we obtain

$$
\alpha(x)=\lim _{\alpha \uparrow \alpha(x)} \underline{\lambda}_{\alpha}=\lim _{\alpha \uparrow \alpha(x)} \varliminf_{n \rightarrow \infty} \hat{q}^{n d_{\alpha}}(x, x)=\varliminf_{n \rightarrow \infty} \lim _{\alpha \uparrow \alpha(x)} \tilde{q}^{n d_{\alpha}}(x, x)=\varliminf_{n \rightarrow \infty} \tilde{q}^{n d(x)}(x, x) .
$$

Similarly we also have $\alpha(x)=\varlimsup_{\lim } \rightarrow \infty \hat{q}^{n d(x)}(x, x)$. Therefore we get theorem.

Further, we need some notations in Yoshida [6, p.42] to describe the ergodic property. Put a path space by $\Omega:=\prod_{n=0}^{\infty} E$ and write a path by $\omega=(\omega(0), \omega(1), \omega(2), \cdots) \in \Omega$. $\mathcal{M}$ denotes the $\sigma$-field generated by all Borel subsets of $\Omega$. We define a map $X_{n}(\omega):=\omega(n)$ for $n \in \mathrm{N}$ and $\omega=(\omega(0), \omega(1), \omega(2), \cdots) \in \Omega$. Then $X_{n}(\omega)$ means a point at time $n$ when the system transits along the path $\omega$. For $y \in E$, the first hitting time of the point $y$ is defined by

$$
\sigma_{\{y\}}(\omega):=\inf \left\{n \geq 1 \mid X_{n}(\omega)=y\right\} \quad \omega \in \Omega,
$$

where the infimum of the empty set is understood to be $+\infty$. For an initial state $x \in E$ and an $\mathcal{M}$-measurable fuzzy set $h \in \mathcal{F}(\Omega)$, we define a fuzzy expectation by

$$
E_{x}(h):=f_{\{\omega \in \Omega: \omega(0)=x\}} h(\omega) \mathrm{d} \tilde{P}(\omega)
$$

where $\tilde{P}$ is the following possibility measure :

$$
\tilde{P}(\Lambda):=\sup _{\omega \in \Lambda} \bigwedge_{n \in N} \tilde{q}\left(X_{n} \omega, X_{n+1} \omega\right) \quad \Lambda \in \mathcal{M}
$$

and $f \mathrm{~d} \tilde{P}$ denotes Sugeno integral (Sugeno [5]). We also put $P_{x}(\Lambda):=E_{x}\left(1_{\Lambda}\right)$ for $\Lambda \in \mathcal{M}$ and $x \in E$. The fuzzy expectation is an extension of the fuzzy transition $\left\{\tilde{q}^{n}\right\}_{n=0}^{\infty}$ and admits stopping times depending on paths. We note that a possibility of transition from $x(\in E)$ to $y(\in E)$ at the $n$th step becomes $P_{x}\left(X_{n}=y\right)=\tilde{q}^{n}(x, y)$. Define the following possibilities :

$$
\tilde{r}^{m}(x, y):=P_{x}\left(\sigma_{\{y\}}=m\right) \quad m=1,2, \cdots ; x, y \in E
$$


and

$$
\gamma(x, y):=\sup _{m \geq 1} \tilde{r}^{m}(x, y)=P_{x}\left(\sigma_{\{y\}}<\infty\right) \quad x, y \in E .
$$

Then, by [7, Proposition 2.1(ii)], we obtain

$$
\gamma(x, y)=\sup _{m \geq 1} \hat{q}^{m}(x, y) .
$$

Theorem 3.2. Let $y \in A^{*}$ and $y \in A_{0}(x)$ such that $d(y)<\infty$. Then there exists a positive integer $d(x, y)$, which divides $d(y)$, and an integer $n(x, y)(0 \leq n(x, y)<d(x, y))$ such that

$$
\lim _{n \rightarrow \infty} \tilde{q}^{n d(x, y)+n(x, y)}(x, y)=\gamma(x, y) \wedge \alpha(y)
$$

Proof. For all $n=1,2, \cdots$, we have

$$
\begin{aligned}
\hat{q}^{n}(x, y) & =\max _{m=1,2, \cdots, n}\left\{\hat{r}^{m}(x, y) \wedge \tilde{q}^{(n-m)}(y, y)\right\} \\
& \leq \sup _{m \geq 1} \hat{r}^{m}(x, y) \wedge \sup _{l \geq 1} \tilde{q}^{l}(y, y) \\
& =\gamma(x, y) \wedge \alpha(y) .
\end{aligned}
$$

Let $\alpha$ satisfy $0<\alpha<\gamma(x, y) \wedge \alpha(y)$. Then $y \in A_{\alpha}(x)$. From Lemma 2.4, there exists a positive integer $N_{x, y, \alpha}$ such that

$$
\hat{q}^{n d_{y, \alpha}+n_{x, y, \alpha}}(x, y) \geq \alpha \quad \text { for all } n \geq N_{x, y, \alpha} .
$$

Together with (3.6), we have

$$
\gamma(x, y) \wedge \alpha(y) \geq \varliminf_{n \rightarrow \infty} \tilde{q}^{n d_{y, \alpha}+n_{x, y, \alpha}}(x, y) \geq \alpha .
$$

We define

$$
d(x, y):=\lim _{\alpha \uparrow \gamma(x, y) \wedge \alpha(y)} d_{y, \alpha} \text { and } n(x, y):=\lim _{\alpha \uparrow \gamma(x, y) \wedge \alpha(y)} n_{x, y, \alpha} .
$$

Then $d(x, y)$ divides $d(y)$ from Lemma 2.2(i) since $\alpha<\alpha(y)$. Letting $\alpha \uparrow \gamma(x, y) \wedge \alpha(y)$ in (3.7), in the same reason as the proof of Theorem 3.1 , we obtain

$$
\varliminf_{n \rightarrow \infty} \hat{q}^{n d(x, y)+n(x, y)}(x, y)=\gamma(x, y) \wedge \alpha(y) .
$$

Similarly, we also have

$$
\varlimsup_{n \rightarrow \infty} \tilde{q}^{n d(x, y)+n(x, y)}(x, y)=\gamma(x, y) \wedge \alpha(y) .
$$

Therefore we get this theorem.

Finally, we give an ergodic theorem regarding $\left\{\hat{q}^{m}(x, y)\right\}_{m=1}^{\infty}$ for the dynamic fuzzy system. By Lemma 2.2, for $y \in A^{*}$ and $y \in A_{0}(x)=\bigcup_{\alpha>0} A_{\alpha}(x)$ such that $d(y)<\infty$, 
there exists a finite number of levels $\left\{\alpha_{i} \mid i=0,1,2, \cdots, k\right\}$ satisfying the following (3.8) and (3.9):

$$
\begin{gathered}
0=\alpha_{0}<\alpha_{1}<\alpha_{2}<\cdots<\alpha_{k}=\alpha(y) \\
d_{y, \alpha}= \begin{cases}d_{y, \alpha_{i+1}} & \text { if } \alpha_{i}<\alpha \leq \alpha_{i+1} \text { for } i(=0,1,2, \cdots, k-2) \\
d(y) & \text { if } \alpha_{k-1}<\alpha<\alpha_{k} .\end{cases}
\end{gathered}
$$

Further, there exists a positive integer $k^{\prime}\left(k^{\prime} \leq k\right)$ satisfying (3.10) and (3.11) :

$$
\begin{gathered}
\alpha_{k^{\prime}-1}<\gamma(x, y) \wedge \alpha(y) \leq \alpha_{k^{\prime}}, \\
d_{y, \alpha}=d(x, y) \quad \text { if } \alpha_{k^{\prime}-1}<\alpha<\alpha_{k^{\prime}} .
\end{gathered}
$$

Then, by replacing $\alpha_{k^{\prime}}$ by $\gamma(x, y) \wedge \alpha(y) \wedge \alpha_{k^{\prime}}$, from Lemma 2.4(i) we have

$$
n_{x, y, \alpha}= \begin{cases}n_{x, y, \alpha_{i+1}} & \text { if } \alpha_{i}<\alpha \leq \alpha_{i+1} \text { for } i\left(=0,1,2, \cdots, k^{\prime}-2\right), \\ n(x, y) & \text { if } \alpha_{k^{\prime}-1}<\alpha<\alpha_{k^{\prime}}\end{cases}
$$

For simplicity, we write $d_{i}:=d_{y, \alpha_{i}}$ and $n_{i}:=n_{x, y, \alpha_{i}}$ for $i=1,2, \cdots, k^{\prime}-1$ and we also write $d_{k^{\prime}}:=d(x, y)$ and $n_{k^{\prime}}:=n(x, y)$.

Theorem 3.3 (ergodic theorem). Let $x, y \in E$ satisfy $y \in A_{0}(x)$ and $d(y)<\infty$.

(i) If $y \notin A^{*}$, then

$$
\tilde{q}^{m}(x, y)=0 \text { for all } m=1,2, \cdots
$$

(ii) If $y \in A^{*}$, then we have the following three cases :

(a) Case of $m \equiv n(x, y)(\bmod d(x, y))$ :

$$
\lim _{n \rightarrow \infty} \hat{q}^{n d(x, y)+n(x, y)}(x, y)=\gamma(x, y) \wedge \alpha(y) .
$$

(b) Let $i=1,2, \cdots, k^{\prime}-1$. Case of $m \not \equiv n_{i+1}\left(\bmod d_{i+1}\right)$ and $m \equiv n_{i}\left(\bmod d_{i}\right)$ : There exists a positive integer $N_{i}$ such that

$$
\tilde{q}^{m}(x, y)=\alpha_{i} \text { for all } m \in \mathbf{J}(i) \text { satisfying } m \geq N_{i},
$$

where $\mathbf{J}(i):=\left\{m \mid m\right.$ is a positive integer satisfying $m \not \equiv n_{i+1}\left(\bmod d_{i+1}\right)$ and $m \equiv$ $\left.n_{i}\left(\bmod d_{i}\right)\right\}$.

(c) Case of $m \not \equiv n_{1}\left(\bmod d_{1}\right)$ :

$$
\tilde{q}^{m}(x, y)=0
$$

Proof. (i) If $y \notin A^{*}$, then $\alpha(y)=0$. By (3.6), we get $\tilde{q}^{m}(x, y)=0$ for all $m=1,2, \cdots$.

(ii.a) is from Theorem 3.2 .

(ii.b) Let $i$ satisfy $1 \leq i<k^{\prime}$. Let $\alpha$ satisfy $\alpha_{i}<\alpha<\alpha_{i+1}$. From Lemmas 2.2(ii) and 2.4 , we have

$$
d_{y, \alpha}=d_{y, \alpha_{i+1}}=d_{i+1} \quad \text { and } \quad n_{x, y, \alpha}=n_{x, y, \alpha_{i+1}}=n_{i+1} .
$$


From (3.11) and Lemma 2.4(i)(ii), there exists a positive integer $N_{i}$ such that

$$
\alpha_{i} \leq \hat{q}^{m}(x, y)<\alpha \quad \text { for all } m \in \mathbf{J}(i) \text { satisfying } m \geq N_{i}
$$

Since $N_{i}$ depends only on $x, y$ and $\alpha_{i+1}$, letting $\alpha \downarrow \alpha_{i}$ in (3.12),

$$
\tilde{q}^{m}(x, y)=\alpha_{i} \quad \text { for all } m \in \mathbf{J}(i) \text { satisfying } m \geq N_{i} .
$$

Therefore we get (ii.b).

(ii.c) Similarly to the proof of (ii.b), for $i=0$, we get

$$
\tilde{q}^{m}(x, y)=\alpha_{0}=0 \text { for all } m \text { satisfying } m \not \equiv n_{1}\left(\bmod d_{1}\right) \text {. }
$$

Thus we obtain this theorem.

\section{Numerical examples}

We consider a one-dimensional numerical example with a one-dimensional state space $E=\mathbf{R}$, where $\mathbf{R}$ denotes the set of all real numbers. Then, $\tilde{q}_{\alpha}(x)$ are bounded closed intervals of $\mathbf{R}(\alpha \in(0,1], x \in \mathbf{R})$ and is written by $\tilde{q}_{\alpha}(x)=\left[\min \tilde{q}_{\alpha}(x)\right.$, $\left.\max \tilde{q}_{\alpha}(x)\right]$, where $\min F(\max F)$ denotes the minimum (maximum resp.) point of a interval $F \subset \mathrm{R}$. We give a fuzzy relation, which is not monotone in the sense of [7], by

$$
\tilde{q}(x, y)=\left(1-\left|x+y^{3}\right|\right) \vee 0, \quad x, y \in \mathbf{R} .
$$

Then $\tilde{q}(x, y)$ satisfies the conditions in Section 2 (see Figure 4.1).

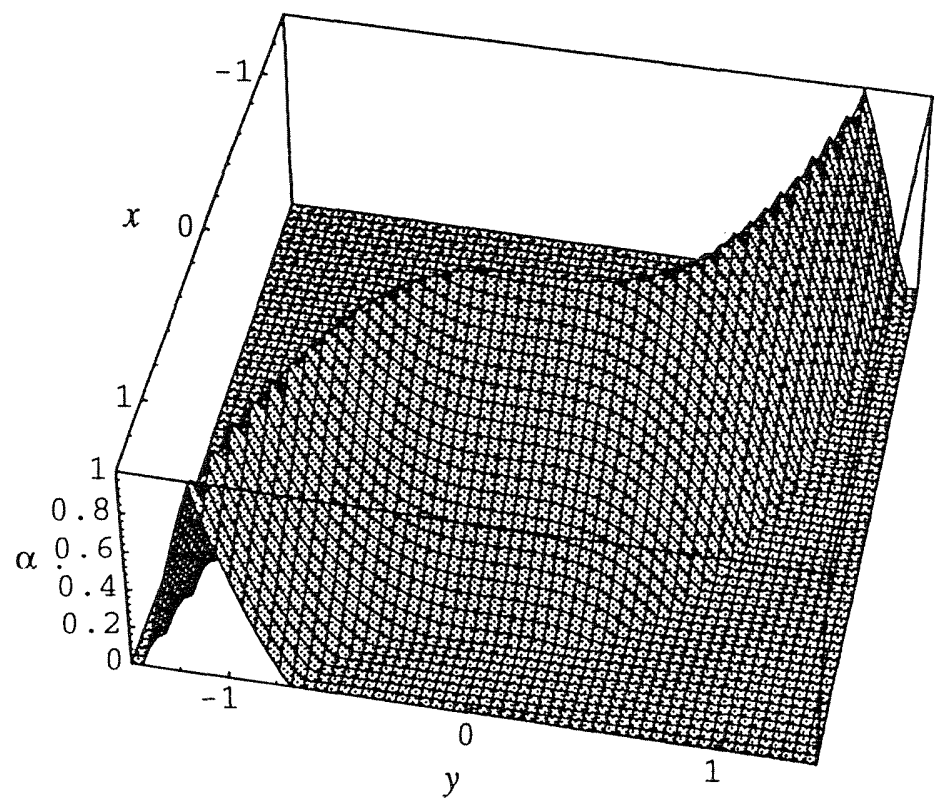

Fig. 4.1: The fuzzy relation $\tilde{q}(x, y)$. 
In this paper, we call a set $\{x \in \mathbf{R} \mid \tilde{q}(x, x)=\alpha\} \alpha$-slice for each $\alpha \in[0,1]$. Figures 4.2 and 4.3 show its 0.7 -slice and 0.5 -slice respectively.

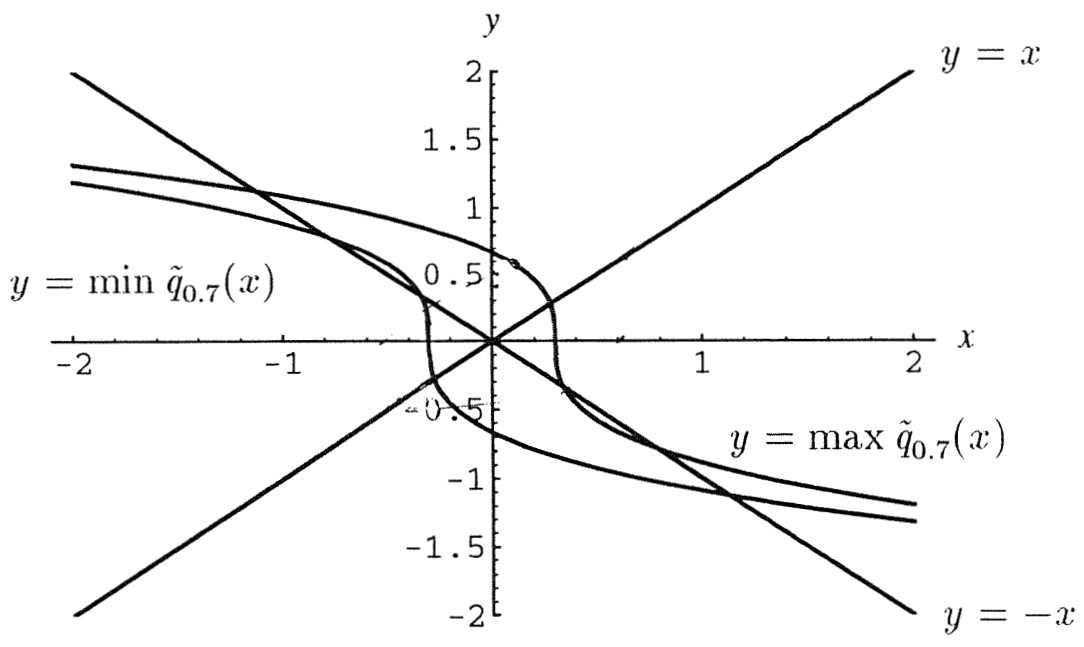

Fig. 4.2. The 0.7-slice $\{(x, y) \mid \tilde{q}(x, y)=0.7\}$.

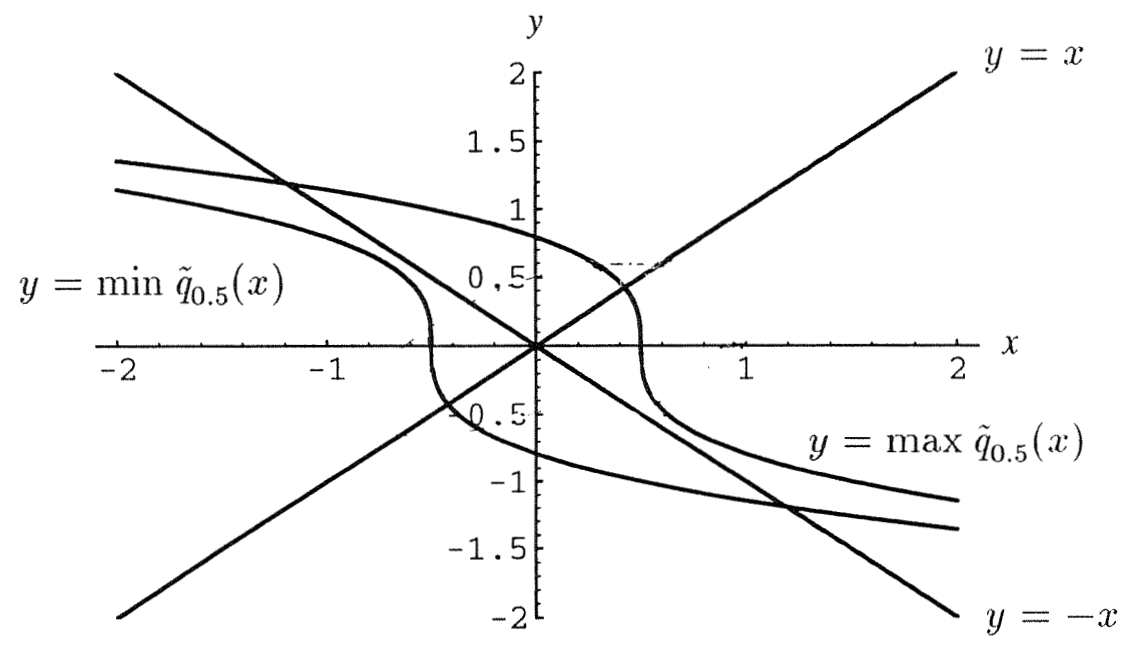

Fig. 4.3. The 0.5 -slice $\{(x, y) \mid \tilde{q}(x, y)=0.5\}$.

First, we consider the cyclic property in Section 2. In this example, we have

$$
\begin{aligned}
F_{\alpha} & :=\left\{y \in \mathbf{R} \mid \sup _{n \geq 1} \tilde{q}^{n}(y, y) \geq \alpha\right\}=\left\{y \in \mathbf{R} \mid \tilde{q}(y, y) \vee \tilde{q}^{2}(y, y) \geq \alpha\right\} \\
& =\left\{x \in \mathbf{R} \mid \min \tilde{q}_{\alpha}(x) \leq-x \leq \max \tilde{q}_{\alpha}(x)\right\} \quad \text { for } \alpha \in(0,1] .
\end{aligned}
$$

Then $F_{0.5}$ is a closed interval. However, $F_{0.7}$ becomes a union of three closed intervals (see Figures 4.2 and 4.3$)$. Therefore we write it by $F_{0.7}=F_{0.7,0} \cup F_{0.7,1} \cup F_{0.7,2}$. Calculating them, we have

$$
\begin{aligned}
& F_{0.7,0} \approx[-1.1254,-0.7865], \\
& F_{0.7,1} \approx[-0.3389,0.3389], \\
& F_{0.7,2} \approx[0.7865,1.1254],
\end{aligned}
$$


and

$$
F_{0.5} \approx[-1.1915,1.1915] .
$$

For $\alpha=0.7, F_{0.7,1}$ and $F_{0.7,0} \cup F_{0.7,2}$ are 0.7 -essential, which is 0.7 -recurrent sets in the sense of Yoshida [7]. $F_{0.7,1}$ is not 0.7-closed. $F_{0.7,0} \cup F_{0.7,2}$ is 0.7-closed, and $F_{0.7,0}$ and $F_{0.7,2}$ are cyclic classes of $F_{0.7,0} \cup F_{0.7,2}$. The periods are

$$
d_{y, 0.7}= \begin{cases}1 & \text { if } y \in F_{0.7,1} \\ 2 & \text { if } y \in F_{0.7,0} \cup F_{0.7,2}\end{cases}
$$

For $\alpha=0.5, F_{0.5}$ is 0.5 -essential and 0.5 -closed. We also have the period $d_{y, 0.5}=1$ for $y \in F_{0.5}$.

Next, we consider the ergodic property in Section 3. In this example, we have

$$
\alpha(y)=\tilde{q}(y, y) \vee \tilde{q}^{2}(y, y)=\left(1-\left|-y+y^{3}\right|\right) \vee 0 \quad \text { for } y \in \mathbf{R} .
$$

Figure 4.4 shows the graph.

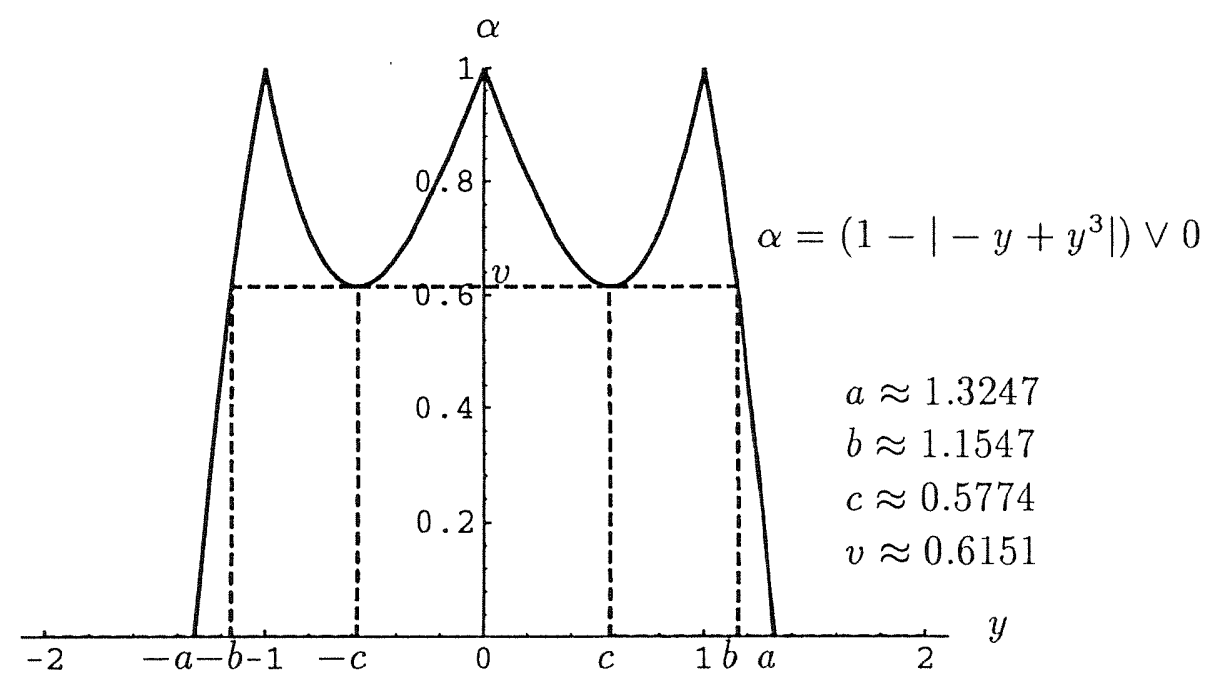

Fig. 4.4. The graph of $\alpha=\alpha(y)$.

Then we put the union of all positive recurrent sets by

$$
(-a, a):=A^{*}=\bigcup_{\alpha>0} F_{\alpha}=\{y \in \mathbf{R} \mid \alpha(y)>0\} \approx(-1.3247,1.3247) .
$$

We also put

$$
b:=\frac{2}{\sqrt{3}} \approx 1.1547, \quad c:=\frac{1}{\sqrt{3}} \approx 0.5774, \quad v:=1-\frac{2}{3 \sqrt{3}} \approx 0.6151 .
$$

From Figure 4.4, we obtain the following three cases (C.1), (C.2) and (C.3) :

(C.1) If $y \notin[-a, a]$, then

$$
\tilde{q}^{m}(y, y)=0 \text { for all } m=1,2, \cdots
$$


(C.2) If $y \in(-b,-c) \cup(c, b)$, we may take

$$
\begin{gathered}
\alpha_{0}=0, \quad \alpha_{1}=v, \quad \alpha_{2}=\left(1-\left|-y+y^{3}\right|\right) \vee 0 \\
d_{y, \alpha}= \begin{cases}d_{1}=1 & \text { if } \alpha_{0}<\alpha \leq \alpha_{1} \\
d_{2}=2 & \text { if } \alpha_{1}<\alpha<\alpha_{2}\end{cases}
\end{gathered}
$$

Then we have the period $d(y)=2$. By Theorem 3.3, we get the following cases :

(a) Case of $m \equiv 0(\bmod 2)$ :

$$
\lim _{n \rightarrow \infty} \hat{q}^{n \times 2}(y, y)=\alpha_{2}=\left(1-\left|-y+y^{3}\right|\right) \vee 0 .
$$

(b) Case of $m \equiv 1(\bmod 2):$ There exists a positive integer $N_{1}$ such that

$$
\tilde{q}^{m}(y, y)=\alpha_{1}=v \quad \text { for all odd } m \text { satisfying } m \geq N_{1} .
$$

(C.3) If $y \in[-a,-b] \cup[-c, c] \cup[b, a]$, we may take

$$
\alpha_{0}=0, \quad \alpha_{1}=\left(1-\left|-y+y^{3}\right|\right) \vee 0,
$$

and $d_{y, \alpha}=d_{1}=1$ if $\alpha_{0}<\alpha<\alpha_{1}$. Then we have the period $d(y)=1$. By Theorem 3.3 , we get

$$
\lim _{m \rightarrow \infty} \tilde{q}^{m}(y, y)=\alpha_{1}=\left(1-\left|-y+y^{3}\right|\right) \vee 0
$$

\section{References}

[1] R.E.Bellman and L.A.Zadeh, Decision-making in a fuzzy environment, Management Sci. Ser B. 17 (1970) 141-164.

[2] K.L.Chung, Markov Chains with Stationary Transition Probabilities (Springer, Berlin, 1960).

[3] M.Kurano, M.Yasuda, J.Nakagami and Y.Yoshida, A limit theorem in some dynamic fuzzy systems, Fuzzy Sets and Systems 51 (1992) 83-88.

[4] D.Revuz, Markov Chains (North-Holland, New York, 1975).

[5] M.Sugeno, Fuzzy measures and fuzzy integral : a survey in M.M.Gupta, G.N.Saridis and B.R.Gaines, Eds., Fuzzy Automata and Decision Processes (North-Holland, Amsterdam, 1977) 89-102.

[6] Y.Yoshida, Markov chains with a transition possibility measure and fuzzy dynamic programming, Fuzzy Sets and Systems 66 (1994) 39-57.

[7] Y.Yoshida, The recurrence of dynamic fuzzy systems, RIFIS Technical Report 80 (1994), Research Institute of Fundamental Information Science, Kyushu University, Japan.

[8] Y.Yoshida, M.Yasuda, J.Nakagami and M.Kurano, A potential of fuzzy relations with a linear structure: The contractive case, Fuzzy Sets and Systems 60 (1993) 283-294.

[9] Y.Yoshida, M.Yasuda, J.Nakagami and M.Kurano, A potential of fuzzy relations with a linear structure : The unbounded case, Fuzzy Sets and Systems 66 (1994) 83-95. 